\title{
The Application of ATMEL SAM3 MCU in State Grid electric power collector
}

\author{
Wang Yanqin ${ }^{1, a}$, Wang Hong ${ }^{2, b}$, Liu Shuang ${ }^{3, c}$ \\ ${ }^{1}$ Department of Industrial and commercial college, Hebei University, Baoding 071002, China \\ ${ }^{2}$ Department of electronics and Information Engineering, Hebei University, Baoding 071002, China \\ ${ }^{3}$ Department of Quality Technology Supervision, Hebei University, Baoding 071002, China \\ alianlianfushi@126.com, ${ }^{b} 176281796 @ q q . c o m,{ }^{c}$ liushuang99@hotmail.com
}

Keywords: ATMEL SAM3N, electric power collector, smart grid

\begin{abstract}
The article describes how to use MCU that the latest low-cost of ATMEL SAM3 series to achieve the ideas and implementation methods of smart grid collector, and elaborates the specific programs from hardware to software implementation process. The schemes has the advantages of low-cost, high-performance, and high-reliability and meets the actual needs of hardware resources, the demanding requirement of cost-performance and reliability in the power industry, and now it has been applied in the National Grid collector.
\end{abstract}

\section{Introduction}

In recent years, with the rapid development of internet technology and semiconductor technology, the traditional power grid has been unable to meet the actual needs of the power industry, the intelligent grid development has become an inevitable trend, and the development of the smart grid has been formed worldwide consensus. From the perspective of technology development and application, experts and scholars around the world generally believe that smart grid is a new modern power grid, which is combined by an advanced sensor measurement technology, information communication technology, analysis and decision-making technology, automatic control technology, energy power technology, and highly integrated grid infrastructure. As the research and development of the smart grid is still in its early stage, and the national circumstances and distribution of resources are also different, the direction and focus of the development of smart grid are not same. There is no uniform and clear definition around the world. According to the current research, the smart grid is to inject new technologies into the grid, including the advanced communication technology, computer technology, information technology, automatic control technology and power engineering technology, to give the grid some kind of artificial intelligence, so that it has a strong strain capacity to become a fully automated power supply network.

\section{The Framework and Composition}

As an important part of the smart grid and the end of the smart grid network, power collector fulfills the work of collecting, gathering and uploading data of the last kilometer. The power collector, as the article describes, is a solution that for the data collection for tenants living in concentrated buildings. The frame diagram of the electric ammeter reading system is shown in Figure 1. 


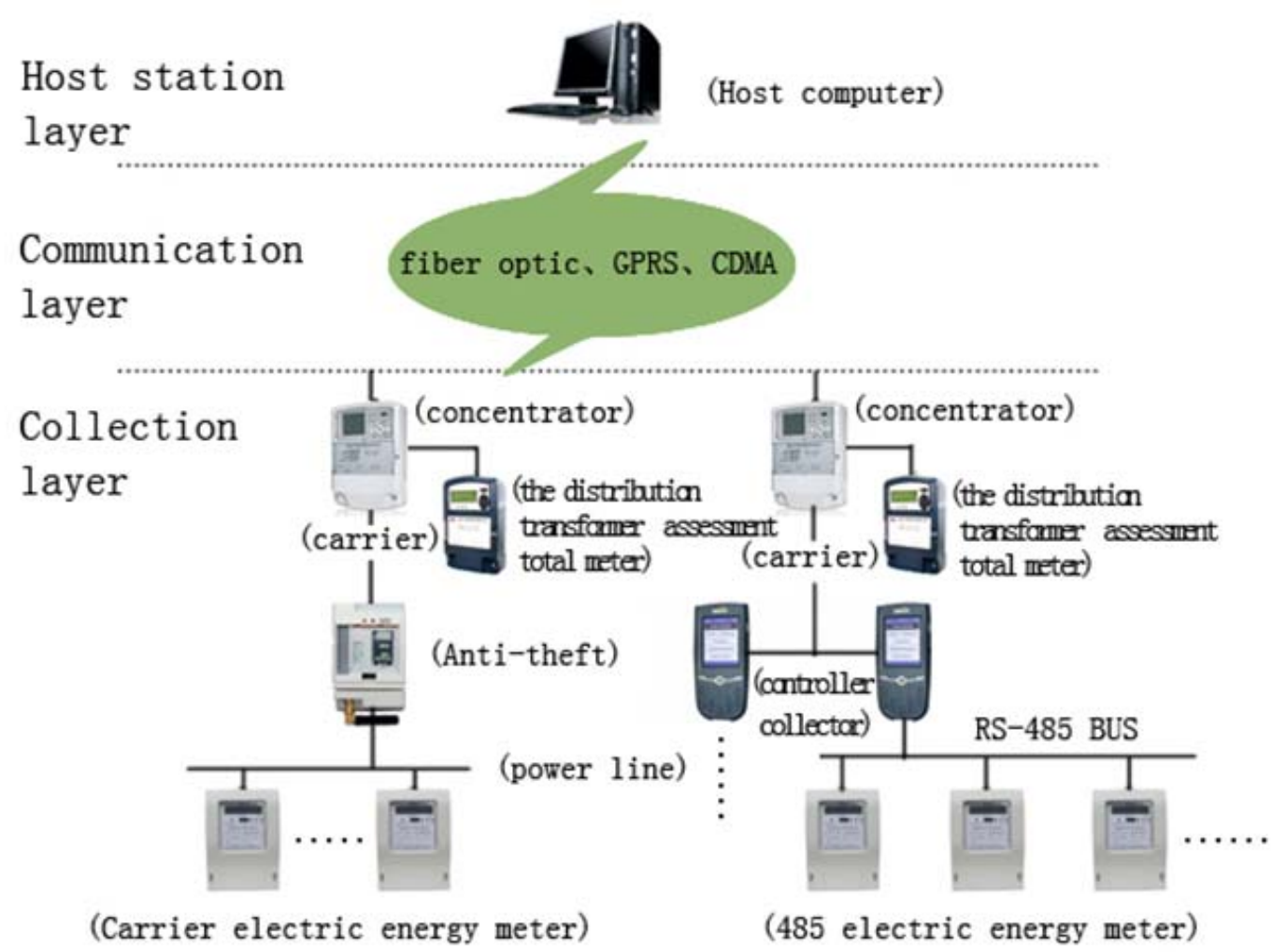

Figure 1.The power meter system framework diagram

Figure 1 clearly summarizes the main structure of electric ammeter reading system. It contains several main components: first, it has a single-phase smart meter(including 485 meter and power line carrier meter)、 power collector、power concentrator、main communication network v the main station 、 and so on.485 single-phase smart meter is already digital, with RS485 communication interface and infrared communication interface, can be achieved automated meter reading by the 485 network, can also be achieved through a handheld console by transcribing data from each separate meter, Carrier meter with carrier capability can directly upload data through the power line to the concentrator not by collector. However, due to the cost of the carrier chip has been high, the single-phase meter is demanding with the cost, therefore restricts the widespread use of the carrier meters. In order to deal with such problems, 485 the use of meter and collector is highly recommended. RS485 with 485 network, generally less than 128 points, aggregate data by collector, and then reported to concentrator by the collector through the carrier. These approaches greatly reduce the cost of the meter, and meet the needs of the power meter. RS485 communication mode is inexpensive in cost, has high reliability, far communication distance, fast speed, strong anti-jamming capability, simple network topology, which is widely used in industrial production and very suitable for single-phase meter of small-scale networking applications. Concentrator summarizes data through carrier and then reports to the master station accessing to wide area network(fiber) over an Ethernet network or via GPRS(or 3G) network reporting through the mobile network to report to the main station(remote or wired network restricted area) finally accesses to WAN.

\section{Functional Requirements of Collector}

This shows that the power collector is a bridge and link between the RS485 meter, which is located at the end of the meter network topology and concentrator. It has the function of collecting and uploading data, and extending the collection capacity. The reason of increasing collectors is because power concentrator generally has extremely great functions and data storage capabilities. However, the user meters are too scattered and in great numbers. The 485 network scale generally cannot be too large, the limit is 256 points. So the direct networking mode of the collector and the meter will be restricted. The concentrator has the ability to manage thousands of meters, but relying 
on the interface of the concentrator itself is unable to complete so many points of the fan-out, thus emerged the concept of collector is introduced.

Collector can be regarded as a way of expanding the interface capability of concentrator, the structure of the collector as shown in figure 2.

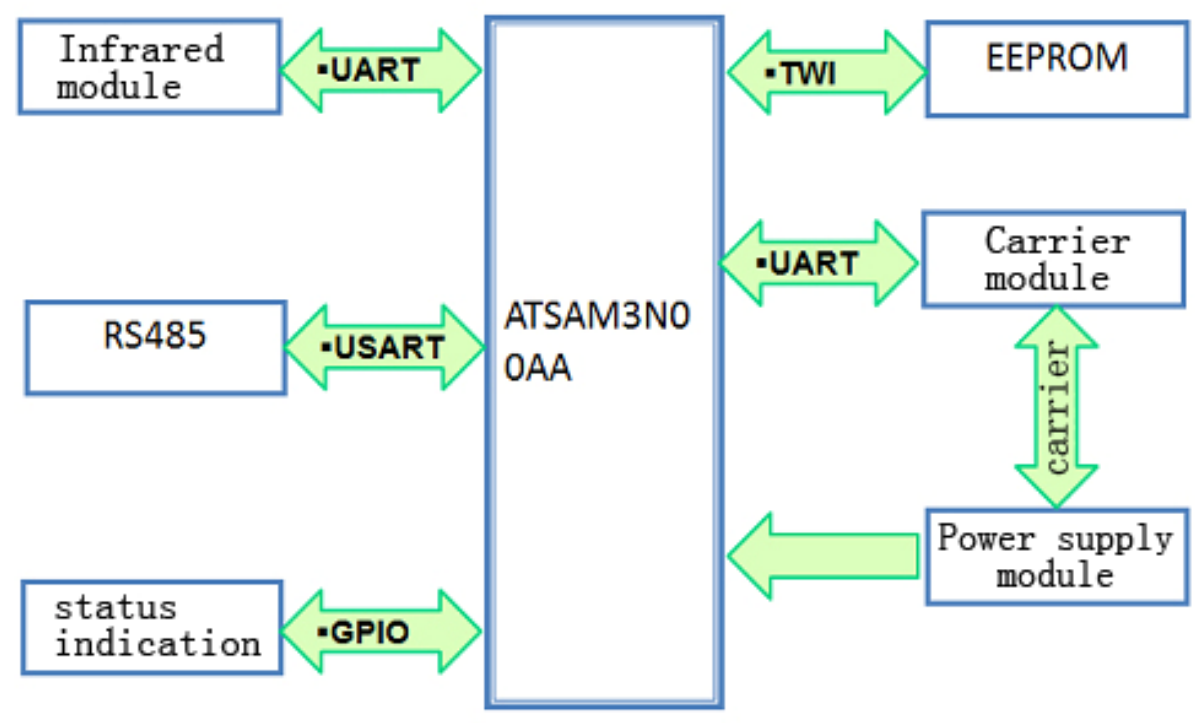

Electricity couector system block diagram

Figure 2 Electricity couector system block diagram

As shown in Figure 2 ,the power collector has the following interfaces RS485 interface(local 485 meter networking); electricity carrier interface(to realize data collecting and uploading); infrared interface(parameter settings, hand-written interface); TWI interface(expanded EEPROM, store configuration parameters), and so on. RS485 is suitable for a small range of networking. Although 485 has good networking characteristics, and is very cheap, long-distance transmission is restricted, since electric power network has wide coverage, local dispersion, and local concentration, so it 485 network itself cannot meet the actual requirement. Power carrier is a very mature technology; transmission data by using the grid constituted by power line can save the cost and achieve the intelligent networking purpose. Now there are lots of mainstream carrier chip manufacturers, multiple carrier program providing manufactures have got the permission of national power grid, so in national power network, power carrier is also an indispensable part of smart grid network. However, due to the relative high cost of the carrier program, adding the carrier function for each meter is restricted, the emergence of the concentrator precisely is a compromise, placing the carrier in concentrators, with carrier communication between concentrator and collection, uploading data, local electricity meter with 485 network, then concentrator uploads the data records that the collector has aggregated to the main station.

Collectors need the RS485 interface, infrared interface (alternate), power line carrier interface. It is the basic needs of small collection, ATMEL SAM3N series, with its characteristics of complete interface, high performance, high reliability, is suitable for this application. The author has selected ATMEL'S ATSAM3NOAA-AU as a collector of master according to the collector's demand. The SAM3N series MCU is the ATMEL cortex M3 core MCUs, belonging to the cortex M3 entry-level products, while positioning as the entry-level but features are not weak, it has very rich peripherals, and 3 USART universal serial port, which just meets our communication interface needs, one as 485, a carrier-ship communication, an infrared, all hardware interfaces increase the reliability of the system, reduce the amount of code, and also reduce the cost.

The following is the introduction to ATMELSAM3N Data manual in English:

Atmel's SAM3N series is a member of a family of Flash microcontrollers based on the high performance 32-bit ARM Cortex-M3 RISC processor. It operates at a maximum speed of $48 \mathrm{MHz}$ 
and features up to 256 Kbytes of Flash and up to 24 Kbytes of SRAM. The peripheral set includes 2x USARTs, 2x UARTs, 2x TWIs, 3x SPI, as well as 1 PWM timer, 6x general purpose 16-bit timers, an RTC, a 10-bit ADC and a 10-bit DAC.

\section{Collector Design}

Cortex M3 is a member of the ARM V7-m family of ARM Company, is also one of the earliest products of Cortex-m series, ARM Cortex-M3 is combined by processor a wide range of breakthrough technologies, only 33,000 kernel performances up to 1.2DMIPS/MHz. The processor also incorporates many tight coupling system peripherals, the system can meet the control demand of industry. The advantages of Cortex are low power consumption, low cost, high performance, a combination of 3 (or 2). The current Cortex kernel products have the traditional MCU industry, 8 MCU 16 MCU brings huge impact, with the new generation of M0 lower cost Cortex MCU production, the future of MCU market change unpredictably.The application of Sam3n in the design has the necessary peripherals, and also has a high cost-effective kernel, a robust ecological environment (third IDE platform and system based on CMISS Driven Architecture), it makes it easy for the design of the hardware system resources allocation, even the bottom of the drive is very mature, the focus of work is transferred to the related work of application software, which shortens the development cycle, greatly reduces the intensity of the code work, because the underlying code is extreme labor- intensive work. Following are the system diagrams.

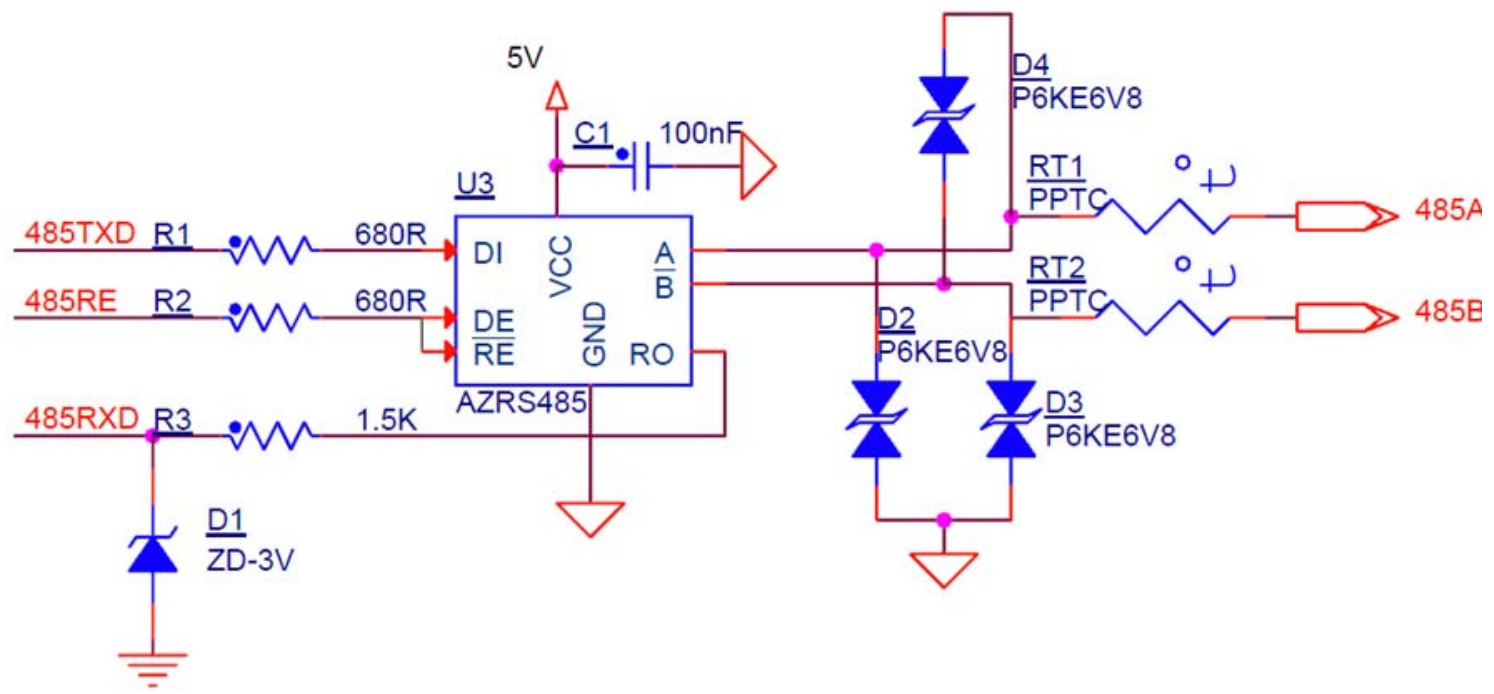

Figure 3 RS485 sending and receiving circuit diagram 


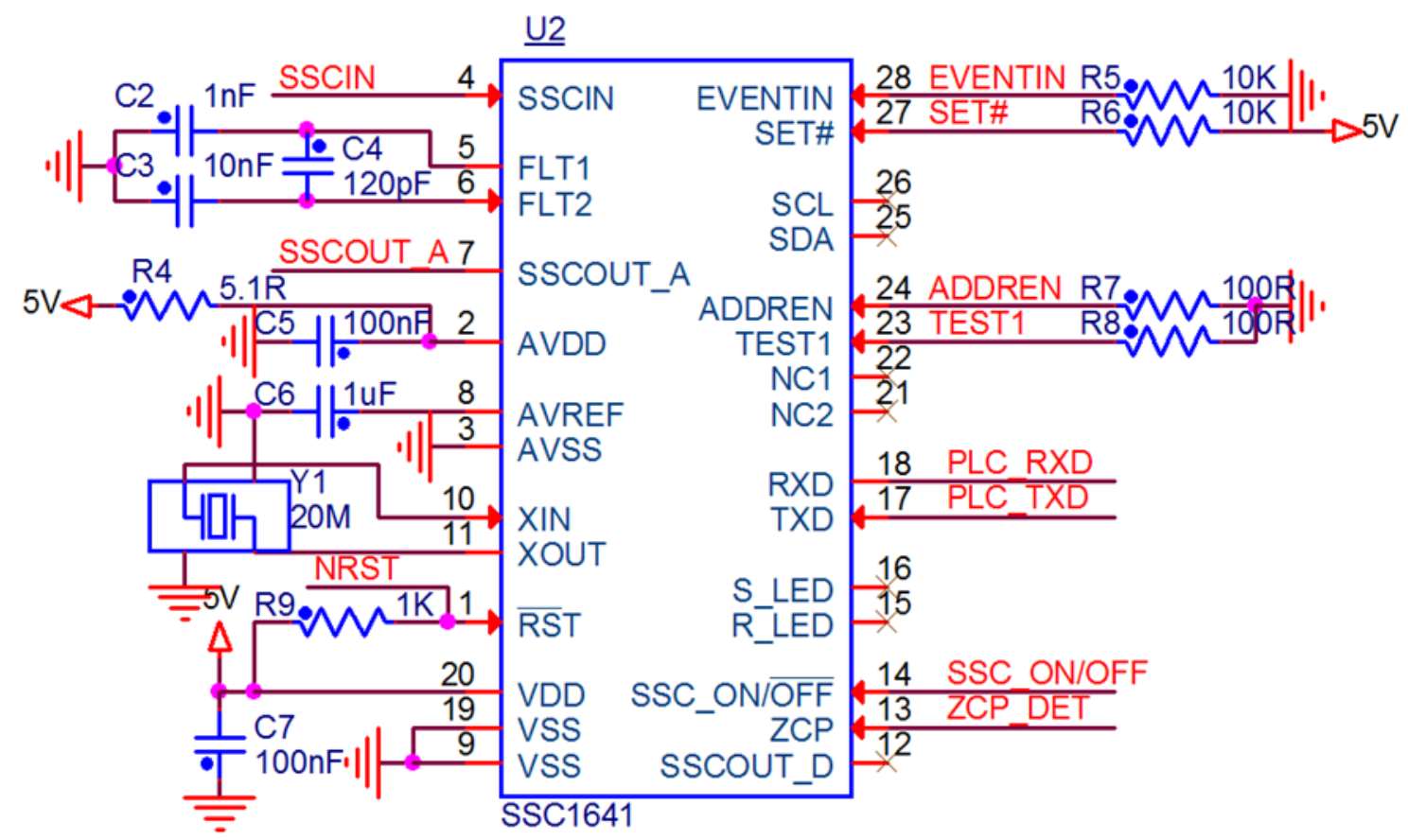

Figure 4 Carrier around chips and circuit diagram

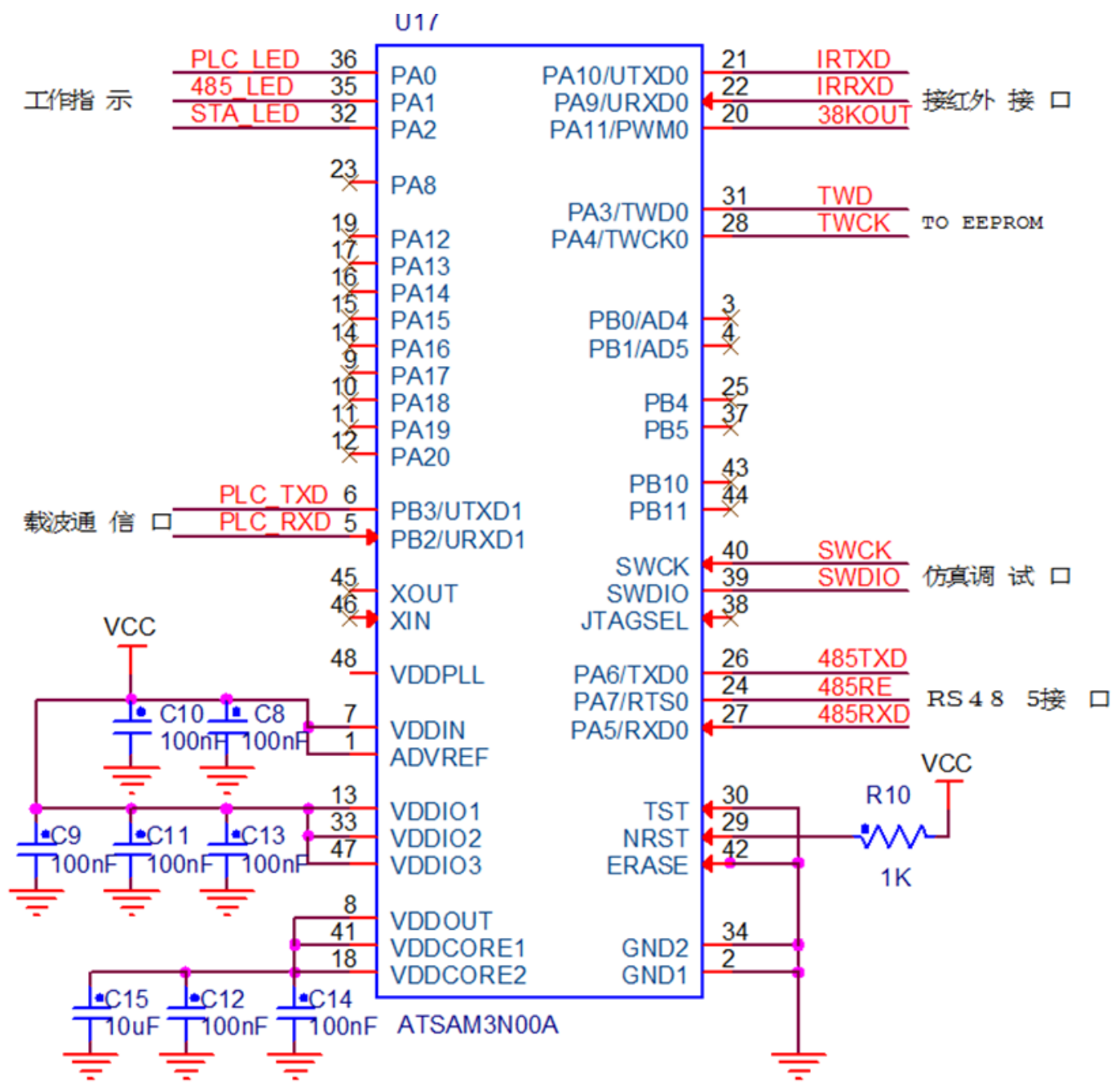

Figure $5 \mathrm{MCU}$ and peripheral circuit diagram

We choose a common ARM company of the cortex M3 kernel, so the IDE environment is very friendly, including ARM's own the Keil MDK platform, an established the compiler vendor IAR, ATMEL corporation also have their own compiled simulation platform studio 6, you can choose 
flexibly according to your preferences. Here we select studio 6 as the development IDE environment, mainly because the studio 6 is designed for the ATMEL SAM3 series, it contains a common driver architecture, which can greatly reduce the workload of coding. For hardware debugging method, there is a variety of hardware debugging method, first, the ATMEL M3series MCU built-in Rom Boot supports SAM BA, which realizes online programming and hardware testing; in addition, by using the IDE environment online real-time simulation through an emulator is achieved.

\section{Conclusion}

Complete the hardware schematic through the hardware block diagram, and then produce the PCB soldering prototype testing. There is something wrong in the SAM BA connection, then it is confirmed that SAM3 16 32KFlash MCU does not support the SAM BA, then instead use the JTAG debug, run studio 6serial port test code, GPIO operation tests are normal, which proves that the hardware is in normal operation, then write the software based on the software flow chart, complete the software written work in accordance with requirements of the statute of the state grid.

Power collector achieves a cost-effective solution of electricity meter, it ensures the performance and reliability of the system, and greatly reduces the cost of the system, it also solves the problem of the shortage of the concentrator fan-out and the high cost of power line carrier, which is the preferred networking solutions of electricity meter.

\section{References}

[1] K.Dostert, Fundamental properties of the Low Voltage Power Distribution Grid Used as a Data Channel, European Transactions on Telecommunications, 2000, pp.297-306

[2] Zhai Mingyue, Measurements and Channel Characteristics of LV Power Line Communications Networks in China, Proceeding of ISPLC 2006, pp.212-216.

[3] ABAD, Ramírez Andrea, Model To Apply AMR Systems In Energy Electromechanical Meter Using Low Voltage Distribution Lines (117 VAC - 60 Hz.) In Colombia, Proceeding of ISPLC 2007, pp. 538-542.

[4] R.J. Ong, J.T. Dawley and P.G. Clem: submitted to Journal of Materials Research (2003)

[5] B.S. Park, D.H.Hyun,Implementation of AMR System Using PowerLine Communication, IEEE, 2002, pp.18-24

[6] A. Lotito, R. Fiorelli, A complete Narrow Band Power Line Communication node for AMR, Proceeding of ISPLC 2007,pp.161-166. 